\title{
A Real-Time Logging System for Managing Multiuser Equipment
}

\author{
X.-Z. Li
}

Nebraska Center for Materials and Nanoscience, University of Nebraska, 855 N. 16th St., N201 NANO, Lincoln, NE 68588

xzli@unl.edu

\begin{abstract}
A real-time logging system (RTLS) has been developed for managing multiuser equipment such as modern electron microscopes that are operated or controlled via a computer. The RTLS is designed to log the usage of the computer and thus the usage of the equipment. The RTLS is a small-size Java program, which is easy to install and use. It has two basic interfaces, a log-in interface and a logbook interface. The first is a semi-transparent shielded layer that covers the computer monitor until a successful log-in occurs, and the second records instrument usage.
\end{abstract}

Keywords: Facility management, real time, logging system, software, RTLS

\section{Introduction}

Scientific research instruments often have many users. Computer software for management of the equipment is required in order to make the usage of the equipment efficient. Basically it includes a calendar for users to make a schedule and a logging system to record the usage. The management software can be an integrated system or an independent system. An integrated system may be convenient, but it could be costly. An independent system is more simple and flexible in that it can include an online calendar and an offline logging system. In addition, many free general-purpose online group calendars can be used for laboratory scheduling, for example, Google Calendar [1], Localendar [2], and Zoho Calendar [3].

This article describes a real-time logging system (RTLS) designed for managing multiuser equipment [1]. It was designed originally for the author's home research center to automatically manage the 24/7 usage of equipment in real time. This RTLS may be helpful for other research groups or facility centers that have a similar need.

\section{Design and Implementation}

We assume that an instrument, for example, a modern electron microscope, is operated through a computer; thus usage of the instrument can be logged as usage of the computer. The RTLS has been designed and written for this purpose. Figure 1 shows a flowchart of the software design for automatically logging usage of the instrument. The three main functions are marked with bold frames. The log-in interface is a shielded layer that covers the computer monitor with input fields for the identification (ID) and the password of a user (Figure 2). The logbook interface records the usage information, which can be displayed or shrunk into iconized size (Figure 3). The registration interface is used to enter/edit the user's information and to generate the user's ID and password (Figure 4). For older instruments without computer control, the RTLS can be used as a logbook only. In this case, the RTLS can be installed in a computer near the instrument.

The RTLS was coded in Java and tested for computers with Microsoft Windows OS (XP and later versions). Figure 2 shows the log-in interface of the RTLS, which is a semi-transparent shielded layer with fields for the user's ID and password. The semitransparent shielded layer allows the parameters of the instrument to be monitored all the time. Figure 3 shows the

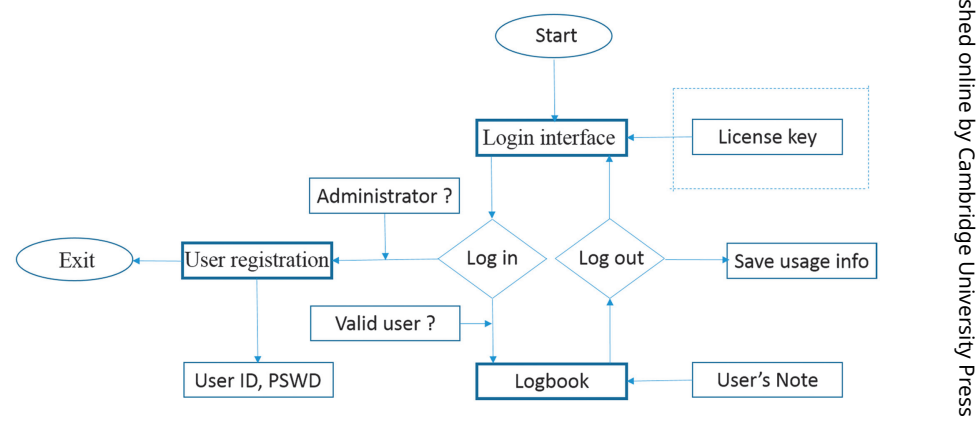

Figure 1: A flowchart of software design for the RTLS. Three main functions are indicated in bold frames.

logbook interface of the RTLS in normal status. The Start time is shown as the time when user logged in. The Current time is a digital clock, which is synchronized with the computer system time. The logbook allows the user to switch between a normal status and an iconized (not shown here) status. When the user logs out, the log-in interface is restored, and the usage information and user's note is saved in formatted text files.

The RTLS may also be used in a registration mode. When the administrator (ID = Admin) logs in, the registration interface displays instead of the logbook, as shown in Figure 4. Thus, new user information can be registered, and the information of registered users can be edited. The RTLS system itself can only be terminated when the administrator logs in.

\section{Installation and Usage}

Availability of the RTLS. An executable landyne_rtls.exe file and a user manual have been packed in .z7 form, and the compressed file is available at this author's webpage [4]. The installation of the RTLS is simple and straightforward. The RTLS package should be decompressed to the landyen_rtls.exe and a user manual in a dedicated folder, for example, c:llandynel rtls $\$. A shortcut can be created and moved to the desktop in Windows OS. Java Runtime Environment (that is, JRE 1.8.0 or up) [5] is required to be pre-installed on the computer.

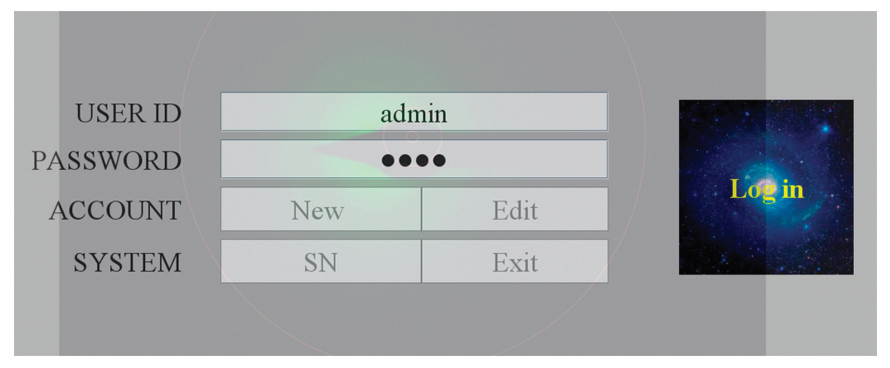

Figure 2: A snapshot of the log-in interface in the real-time logging system. A semitransparent layer in gray is on top of the computer monitor. This is the central part of the monitor. For log-in, users are required to fill in their ID and password. 


\section{June $3-8$,}

\section{LEHIGH}

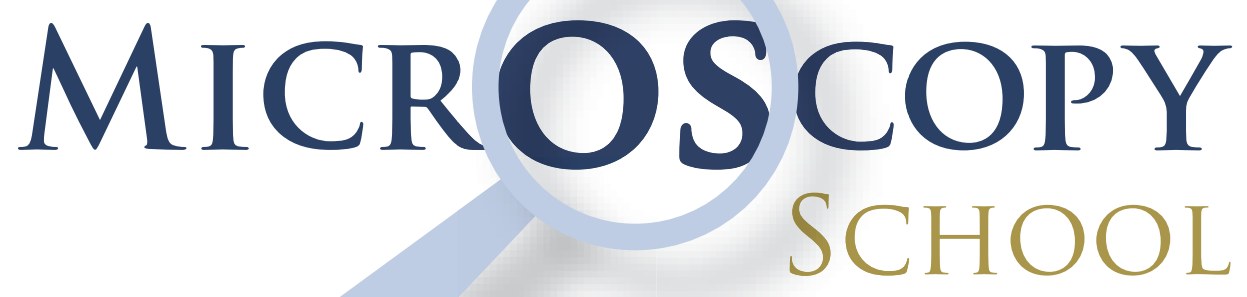

Lehigh University, Bethlehem, PA USA

\section{MAIN COURSES}

SCANNING ELECTRON MICROSCOPY AND X-RAY MICROANALYSIS

June 4-8

\section{SPECIALIZED COURSES}

FOCUSED ION BEAM (FIB): Instrumentation and Applications June 4-8

PROBLEM SOLVING:

Interpretation and Analysis of SEM/EDS/EBSD Data

June 4-8
INTRODUCTION TO SEM AND EDS FOR THE NEW OPERATOR June 3

For more information, contact:

Sharon Coe | 610.758.5133 |

sharon.coe@lehigh.edu

QUANTITATIVE X-RAY

MICROANALYSIS:

Problem Solving using EDS and WDS Techniques

June 4-8

SCANNING TRANSMISSION

ELECTRON MICROSCOPY:

From Fundamentals to Advanced Applications June 4-8
Register and pay in full by April 13 to receive an early bird discount.
48 YEARS OF EX CELLENCE 


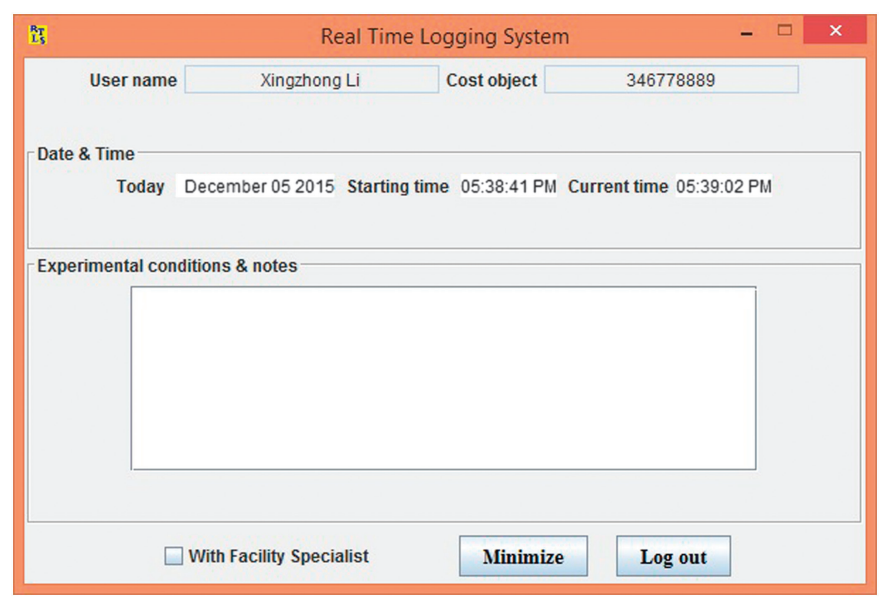

Figure 3: A snapshot of the logbook interface in the real-time logging system. This dialog box shows the starting time and the current. Sample information and experimental conditions can be stored in the log file when the user logs out.

Administrative setup. A detailed description of how to use the RTLS is provided in the user manual. A brief description is given here, with reference to the flowchart in Figure 1. When the software first starts up, the ACCOUNT (New and Edit buttons) and SYSTEM (SN and Exit buttons) are in an inactive status (see Figure 2). These buttons are only accessible by the administrator with a valid password. At the log-in interface, the basic operations in administration are the following: (i) to retrieve the serial number with the SN button, an SN dialog box pops out with the info on the current hard disk and the serial number (SN); (ii) to manage accounts when the New or Edit button is clicked, a User Info dialog box pops up with a blank account (Figure 4). The administrator may create a new account (or an existing account may be edited) by filling in the fields and clicking Save; and (iii) only the administrator may exit the RTLS system with the Exit button. A user's ID and password is generated automatically for each new or modified account. Each user may have multiple accounts if needed.

Use of the RTLS. The RTLS is accessed simply by logging in and then logging out. When a valid ID and password are used to log in, the shield interface will be replaced with the logbook interface, and the user may use the computer to control the equipment. The logbook interface can be set in either iconized status or in normal status to check the experimental time and to write notes. When the user logs out, the information about the user and the usage of the equipment is automatically saved in ASCII text format and

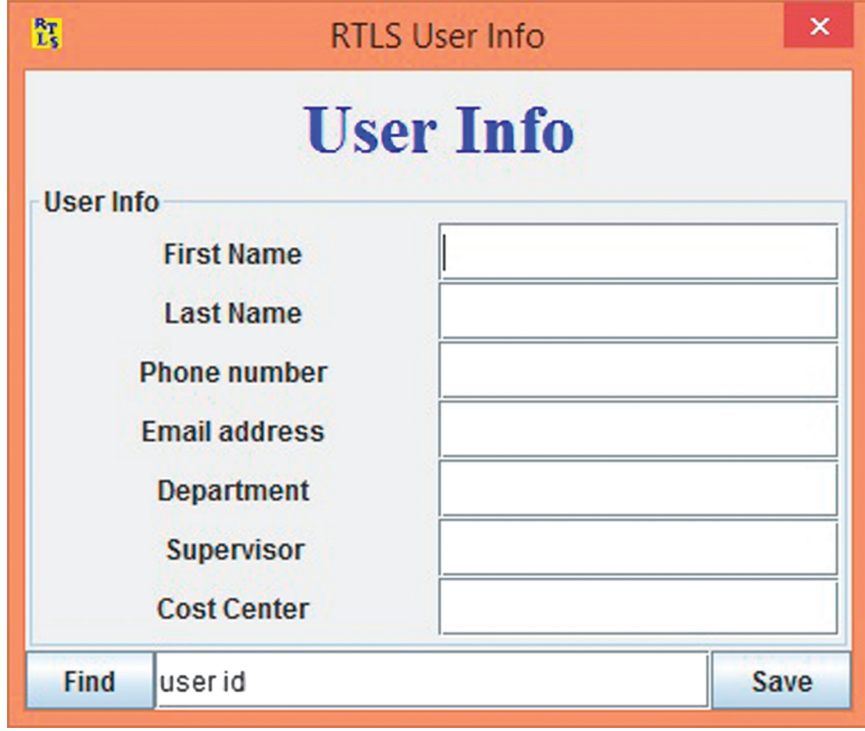

Figure 4: A snapshot of the registration interface in the real-time logging system. The information required for a new user is input here. It will be retrieved when a registered user logs in.

can be loaded into Microsoft Excel for reporting purposes. The $\log$-in interface is then ready for the next user. Table 1 shows an example list of registration data, and Table 2 shows an example list of instrument and service data.

\section{Application Examples}

The RTLS software has been installed in the author's home research center for managing several instruments since 2015, and subsequently it was installed in the electron microscopy facility of Youngstown State University $(\mathrm{OH})$. Two application examples are described here to indicate features of the RTLS.

For an FEI (now Thermo Scientific) Osiris scanning/ transmission electron microscope (S/TEM) with EDS, it is essential that the status of the microscope (for example, liquid nitrogen level and vacuum) can be monitored constantly in real time. The semi-transparent shield of the RTLS is sufficient for this purpose, as shown in Figure 5.

For an FEI NanoSEM scanning electron microscope (SEM) with an Oxford energy dispersive $\mathrm{x}$-ray spectroscopy (EDS) system, it may useful to record the usage of the SEM and the EDS separately. For example, there may be times when the SEM could be booked for just imaging, and the EDS system could

Table 1: Example list of registration data.

\begin{tabular}{|l|l|l|l|l|l|}
\hline User & Email & Phone & Department & Supervisor & Cost Number \\
\hline Ackerkey Smith & asmith@gmail.com & 402472 ACKE & Physics & Thompson, Noah & 2511230056723 \\
\hline Babak Johnson & bjohnson@gmail.com & 402472 BABA & Chemistry & Anderson, Liam & 2511230012341 \\
\hline Caden Williams & cwilliams@gmail.com & 402472 CADE & Engineer & Harris, Mason & 2511230033001 \\
\hline
\end{tabular}

Table 2: Example list of instrument and service data.

\begin{tabular}{|l|l|l|l|c|c|}
\hline Date & User & Supervisor & Cost Number & Instrument (Hour) & Service (Hour) \\
\hline 6/15/2017 & Ackerkey Smith & Thompson, Noah & 2511230056723 & 3.0 & 3.0 \\
\hline 6/16/2017 & Babak Johnson & Anderson, Liam & 2511230012341 & 3.5 & 0.0 \\
\hline 6/17/2017 & Caden Williams & Harris, Mason & 2511230033001 & 4.0 & 2.0 \\
\hline
\end{tabular}




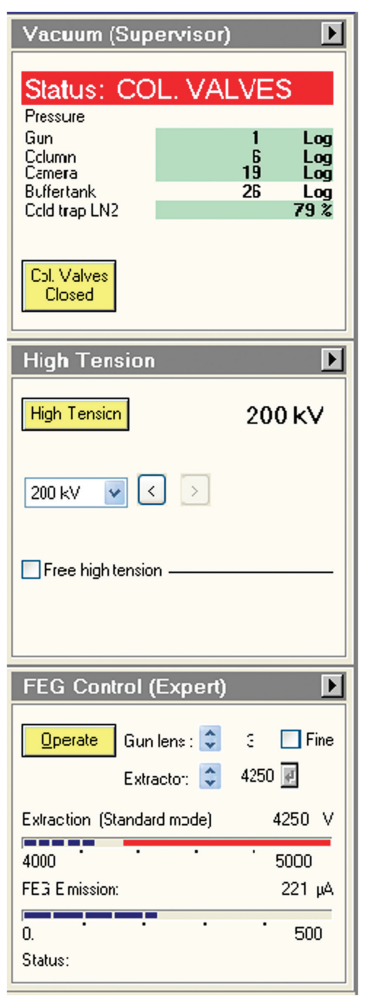

Figure 5: Microscope information is viewable even with the semitransparent layer in gray on top of the computer monitor. For clarity, the gray layer is removed here. be booked separately for detailed spectrum image analysis. The small program size of the RTLS allows it to be installed on both the SEM computer and the EDS computer.

\section{Conclusion}

A real-time logging system, RTLS, has been developed for managing multiuser equipment. The software is a Java logging system, which is easy to install and use. It features two basic interfaces: a log-in interface and a logbook interface. The first one is a semi-transparent shielded layer for sign-in, and the second one records the usage information. The software can be used as a practical tool in a research group or facility center to manage multiuser equipment.

\section{Acknowledgment}

The author would like to thank the editor for his valuable comments and suggestions for the improvement of this article.

\section{References}

[1] Google Calendar, https://calendar.google.com (accessed September 11, 2017).

[2] Localendar, https://localendar.com (accessed September 11, 2017).

[3] Zoho Calendar, https://www.zoho.com/calendar (accessed September 11, 2017).

[4] University of Nebraska-Lincoln, "Software for Electron Diffraction Analysis," http://www.unl.edu/ncmn-cfem/ xzli/computer-programs (accessed September 11, 2017).

[5] Oracle, Java, https://java.com/en/download (accessed September 11, 2017).

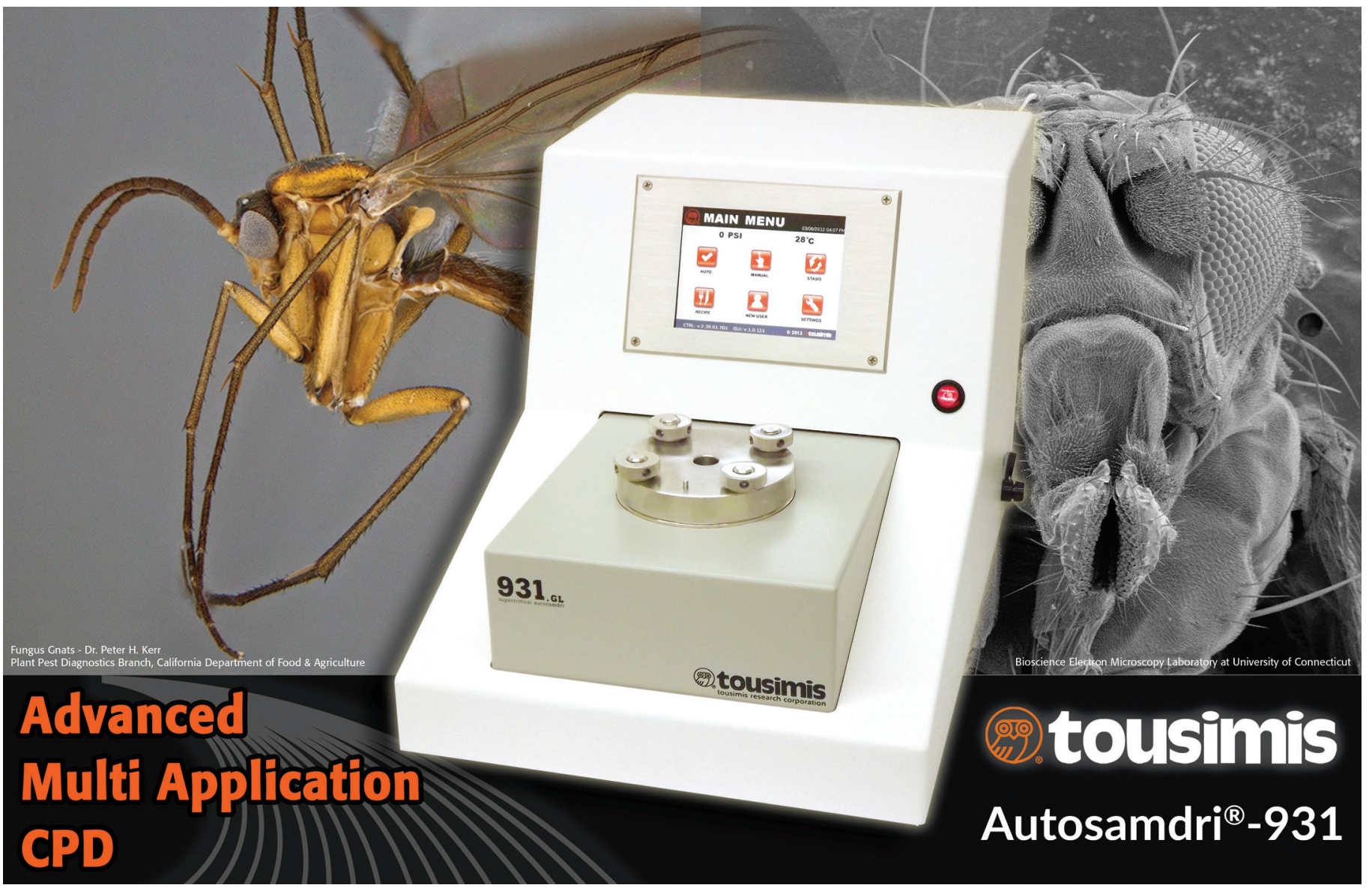

